\title{
Redistribution of GFAP and $\alpha B$-crystallin after thermal stress in C6 glioma cell line
}

\author{
Wei-Chia Tseng, Kuo-Shyan Lu, Wen-Ching Lee \& Chung-Liang Chien* \\ Department of Anatomy and Cell Biology, College of Medicine, National Taiwan University, No. 1, \\ Section 1, Jen-Ai Road, Taipei, 100, Taiwan \\ Received 17 January 2006; accepted 25 April 2006 \\ (C) 2006 National Science Council, Taipei
}

Key words: $\alpha \mathrm{B}$-crystallin, C6 cell, GFAP, glia, heat shock

\begin{abstract}
Summary
Some intermediate filament (IF) proteins expressed in the development of glia include nestin, vimentin, and glial fibrillary acidic protein (GFAP). However, GFAP is the major intermediate filament protein of mature astrocytes. To determine the organization of GFAP in glial cells, rat GFAP cDNA tagged with enhanced green fluorescent protein (EGFP) was transfected into the rat C6 glioma cell line. After selection, two stable C6-EGFP-GFAP cell lines were established. Stable C6-EGFP-GFAP cell lines with or without heat shock treatment were analyzed by immunocytochemistry, electron microscopy, and Western blot analysis. In the transient transfection study, EGFP-GFAP transiently expressed in C6 cells formed punctate aggregations in the cytoplasm right after transfection, but gradually a filamentous structure of EGFP-GFAP was observed. The protein level of nestin in the C6-EGFP-GFAP stable clone was similar to that in the pEGFP$\mathrm{C} 1$ transfected $\mathrm{C} 6$ stable clones and non-transfected C6 cells, whereas the level of vimentin was reduced in Western blotting. Interestingly, the expression level of small heat shock protein $\alpha$ B-crystallin in C6-EGFPGFAP cells was also enhanced after transfection. Immunostaining patterns of C6-EGFP-GFAP cells showed that GFAP was dispersed as a fine filamentous structure. However, after heat shock treatment, GFAP formed IF bundles in C6-EGFP-GFAP cells. In the meantime, $\alpha$ B-crystallin also colocalized with IF bundles of GFAP in C6-EGFP-GFAP cells. The heat-induced GFAP reorganization we found suggested that small heat shock protein $\alpha \mathrm{B}$-crystallin may play a functional role regulating the cytoarchitecture of GFAP.
\end{abstract}

\section{Introduction}

Glial fibrillary acidic protein (GFAP) was first characterized as the major intermediate filament protein of mature astrocytes in the central nervous system (CNS) [1, 2]. GFAP, one of the type III intermediate filament proteins, is an acidic protein with a molecular weight of approximately $50 \mathrm{kDa}$ $[3,4]$. In mammalian development, nestin and vimentin are the main IF proteins in immature

*To whom correspondence should be addressed. Fax + 886-22391-5292; E-mail: clc@ha.mc.ntu.edu.tw astroglial cells, whereas maturing and adult astrocytes contain vimentin and GFAP [5]. It has been reported that nestin production is resumed, and vimentin and GFAP expression is up- regulated in activated astrocytes in reactive gliosis induced by trauma, tumor growth, or neurodegenerative diseases [6, 7]. GFAP is also expressed in tumors derived from astrocytic lineage but malignant astrocytomas have a lower number of GFAPpositive cells than their benign counterparts and normal brain tissue [4]. The function of GFAP remains incompletely understood even though recent findings suggest GFAP involvement in the 
long-term maintenance of the brain architecture, proper function of the blood-brain barrier, and modulation of some neuronal functions by astrocytes [8-11]. Suppression by GFAP antisense RNA in U251 astrocytoma cells can inhibit the process elongation in response to neurons [12]. However, GFAP-null mice show normal astrocytes with process elongation, suggesting that the functional compensation occurs among intermediate filaments $[8,10,13,14]$.

$\alpha \mathrm{B}$-crystallin, a structural component of the vertebrate lens, belongs to a family of small heat shock proteins (HSPs) [15]. $\alpha \mathrm{B}$-crystallin is also found expressed constitutively in non-lenticular tissue such as skeletal or cardiac muscle and kidney [16-18]. This small HSP is inducible in many different cell types by several kinds of physiological stress $[19,20]$, and acts as a molecular chaperone by preventing heat-induced protein aggregation in vitro [21]. It was also reported that $\alpha \mathrm{B}$-crystallin can interact with intermediate filament in muscle, the lens, and astrocytes [22-25], and inhibits the intermediate filament protein assembly in vitro $[23,25]$. Furthermore, in vitro study showed that adenovirus mediated $\alpha \mathrm{B}$ crystallin cDNA transfection can disaggregate the GFAP inclusions in astrocytes [15].

Since GFAP plays an important role in glial development, tumor formation and Alexander disease $[4,26]$, we demonstrated the distribution and reorganization of IFs by overexpression of EGFP-tagged GFAP in C6 cells. The correlation between GFAP and small heat shock protein $\alpha \mathrm{B}$ crystallin was monitored after heat-shock treatment; the role of GFAP together with other intermediate filament proteins in glial cells was also examined in this study.

\section{Materials and methods}

\section{Cloning of the EGFP-GFAP construct}

Rat full-length glial fibrillary acidic protein (GFAP) cDNA (2.7 kb), was obtained from a BamHI fragment of pS65T-GFAP-FL construct. Bam HI fragment of rat GFAP cDNA was subcloned into the BamHI site of pEGFP-C1 (Clontech, Palo Alto, CA, USA) to obtain the in-frame coding sequence with EGFP. The junction for the EGFP-GFAP fusion protein was confirmed by
DNA sequencing. The final construct was named pEGFP-GFAP.

\section{Cell culture}

The rat C6 glioma cells were cultured in Dulbecco's modified Eagle's medium supplemented with $10 \%$ fetal bovine serum (FBS), $1 \%$ antibiotic-antimycotic, and 1\% MEM's non-essential amino acid solution (Gibco, Gaithersburg, MD). Cells were grown on cell culture dishes (Corning, Corning, NY, USA) or flasks (TPP, Trasadingen, Switzerland). All cultures were maintained in a humidified chamber with $5 \% \mathrm{CO}_{2}$ at $37{ }^{\circ} \mathrm{C}$.

\section{DNA transfection and selection}

One day before transfection, cultured cells were trypsinized and plated on glass coverslips $(18 \times 18 \mathrm{~mm})$ in $60 \mathrm{~mm}$ cell culture dishes for transient transfection, or without coverslips for stable transfection. Transfections were performed using Lipofectamine reagent (Invitrogen, Carlsbad, CA, USA) according to the manufacturer's protocol. In most experiments, $3 \mu \mathrm{g}$ plasmid DNA was used for each $60 \mathrm{~mm}$ cell culture dish of cells. The Lipofectamine reagent was diluted with DMEM (Gibco, Gaithersburg, MD) at 1:10, and the plasmid DNA was mixed with the diluted Lipofectamine/D-MEM solution. After cells were rinsed with $1 \times$ D-PBS, $100 \mu \mathrm{l}$ of the DNA/ Lipofectamine/D-MEM mixture was added to each cell culture dish filled with $3 \mathrm{ml}$ D-MEM. Cells were incubated for $18 \mathrm{~h}$ at $37^{\circ} \mathrm{C}$, and then the medium was changed to the respective growth medium. For stable transfection, neomycin analogue G418 (800 $\mu \mathrm{g} / \mathrm{ml}$, Gibco) was added to the culture for selection. After 12-days selection with G418, surviving C6 cells colonies with green fluorescence were selected and picked up under an inverted fluorescence microscope (DM IRBE $\mathrm{HC}$, Leica, Wetzlar, Germany). In addition to the experimental control pEGFP-C1 stable cell clones, two independent pEGFP-GFAP stable cell clones with strong fluorescence were isolated for further studies.

\section{Antibodies}

The following primary antibodies were commercially obtained and applied in this study: (1) mouse 
monoclonal antibodies to nestin (N17220, BD Transduction Laboratories, Lexington, KY, USA), vimentin and GFAP (V5255 and G3893, Sigma, St. Louis, MO, USA), $\beta$-actin (ab6276, Abcam, Cambridge, UK); and (2) rabbit polyclonal antibody to $\alpha \mathrm{B}$-crystallin (sc-22744, Santa Cruz, Santa Cruz, CA, USA)

\section{Western blot analysis}

Confluent $100 \mathrm{~mm}$ cell culture dishes of C6 cells were washed twice in phosphate buffered saline (PBS). To each dish $0.5 \mathrm{ml}$ lysis buffer $[1 \%(\mathrm{v} / \mathrm{v})$ Triton X-100, $150 \mathrm{mM} \mathrm{NaCl}, 1 \mathrm{mM}$ EDTA, $10 \mathrm{mM}$ Tris- $\mathrm{HCl}(\mathrm{pH} 7.5)$ with proteinase inhibitors $(1 \mathrm{mM}$ PMSF, $10 \mu \mathrm{g} / \mathrm{ml}$ pepstatin A, $10 \mu \mathrm{g}$ / $\mathrm{ml}$ leupeptin)] was added. Cells were harvested from the dish by cell scraper and centrifuged at $14,000 \mathrm{~g}$ for $15 \mathrm{~min}$. The supernatant was collected and the pellet was dissolved with SDS-Urea buffer. The protein concentration was measured by a BCA protein quantification kit (Pierce, Rockford, IL, USA). Lysates containing $20 \mu \mathrm{g}$ of protein were electrophoresed in $12 \%$ SDS polyacrylamide gel and subsequently transferred to nitrocellulose papers for Western blotting. Blots were first blocked in 5\% skim milk/TBS for $2 \mathrm{~h}$ at room temperature, and then incubated with antibodies against nestin (1:500), vimentin (1:500), GFAP (1:500), $\alpha \mathrm{B}$-crystallin (1:250), and $\beta$-actin $(1: 500)$ in $5 \%$ skim milk/Tris buffered saline (TBS) at $4{ }^{\circ} \mathrm{C}$, overnight. After washing in TBS-T [TBS with $0.05 \%$ (v/v) Tween 20], blots were incubated with horseradish peroxidase-conjugated goat antimouse or goat anti-rabbit secondary antibodies in a dilution of 1:2000 (AP124P and AP132P, Chemicon, Temecula, CA, USA) at room temperature for $1.5 \mathrm{~h}$. After washing in TBS-T six times, Western blotting luminol reagent kits (Santa Cruz, Santa Cruz, CA, USA) and BioMax films (Kodak, Rochester, New York, USA) were used for detection.

\section{Immunocytochemistry}

Two to eight days after transfection with EGFPGFAP expression vectors, cells were fixed with ice-cold methanol at $-20{ }^{\circ} \mathrm{C}$ for $10 \mathrm{~min}$. Subsequently, cells were rinsed three times in PBS after fixation. After rinses in PBS, cells were permeabilized in PBS containing $0.2 \%(\mathrm{v} / \mathrm{v})$ Nonidet P-40
(NP-40) for $7 \mathrm{~min}$. All primary antibodies were applied overnight at $4{ }^{\circ} \mathrm{C}$ and then rinsed five times for $3 \mathrm{~min}$ each with PBS. For the indirect immunofluorescence detection, tetramethyl rhodamine isothiocyanate (TRITC) or fluorescein isothiocyanate (FITC)-conjugated secondary antibodies were applied for $1 \mathrm{~h}$ at room temperature. Cells were also labeled with fluorescent Hoechst 33342 in a dilution of 1:1000 (Sigma) together with secondary antibodies. After five more rinses in PBS, cells were mounted in crystal mount (Biomeda, Foster City, CA, USA) and analyzed under a TCS SP2 laser confocal microscope (Leica).

\section{Live cell imaging}

Live cell imaging was done in both C6-EGFP and C6-EGFP-GFAP stable clone. Briefly, the cells were trypsinized and replated on glass coverslips $(18 \times 18 \mathrm{~mm})$ in $6 \mathrm{~cm}$ Petri dishes. One day after plating, the cells were rinsed three times with PBS. Then coverslips were picked up and mounted in PBS on glass slides. All images were analyzed and recorded by fluorescent microscope (DMR, HC, Leica) with a D1 $\times$ digital camera and Nikon Capture 4.0 (Nikon, Tokyo, Japan).

\section{Heat shock treatment}

Cells were plated on cell culture dishes with or without glass coverslips, and allowed to attach overnight. These cell cultures were then subjected to heat shock treatment at $42{ }^{\circ} \mathrm{C}$ for $1.5 \mathrm{~h}$ followed by $16 \mathrm{~h}$ recovery at $37^{\circ} \mathrm{C}$ [25]. Cells were then processed for immunocytochemistry and electron microscopy as well as for Western blot analysis.

\section{Immunocytochemistry and statistical analysis}

Cells with or without heat shock treatment were first stained with GFAP antibody and Hoechst 33342 for nucleus staining. In each case, we randomly chose three fields under a $40 \times$ object lens of fluorescent microscope (DMR, Leica). In each field, 300 cells labeled with Hoechst 33342 were counted and then GFAP bundle-bearing cells were counted in the same population. A statistical analysis was performed by Sigma-Plot software (SPSS, Chicago, IL, USA). 


\section{Electron microscopy}

Cells were plated on cell culture dishes with $13-\mathrm{mm}$ diameter plastic coverslips (Electron Microscopy Sciences, Hatfield, PA, USA). After plating overnight, cells were fixed with $4 \%$ paraformaldehyde and $1 \%$ glutaraldehyde in phosphate buffer at $4{ }^{\circ} \mathrm{C}$ overnight, then rinsed with $0.2 \mathrm{M}$ cacodylate buffer three times. Cells were post-fixed with $1 \%$ osmium tetroxide in $0.1 \mathrm{M}$ cacodylate buffer and dehydrated in a graded series of ethanol $(75 \%$, $85 \%, 95 \%$, and $100 \%$ ), and embedded in an Epon-araldite mixture. After the resin had solidified, the plastic culture dish was broken up to release the resin containing the embedded cells. Specimens were sectioned with a Diatome diamond knife on a Rechert Ultracut E ultramicrotome (Leica, Wetzlar, Germeny). Ultrathin sections were stained with uranyl acetate and lead citrate and viewed with a H-7100 electron microscope (Hitachi, Tokyo, Japan). All images were taken by an ORCA-ER CCD digital camera system (Advanced Microscopy Techniques, Danvers, MA, USA).

\section{Results}

Immunocytochemical patterns of pEGFP-GFAP transiently transfected C6 cells

To determine the relationship between GFAP and other IF proteins in C6 cells, cells transiently transfected with pEGFP-GFAP were immunostained with nestin, vimentin, and GFAP antibodies. We examined the organization of EGFPtagged GFAP and other IF proteins 2, 4, and 8 days after transfection. On day 2, EGFP-tagged GFAP was organized into punctate aggregations by itself (Figure 1A), whereas filamentous structures of nestin and vimentin could be found in the cytoplasm of both transfected and non-transfected cells (Figure 1B, C). On days 4 and 8, EGFPtagged GFAP green fluorescence could be matched up with TRITC red fluorescence which demonstrated anti-GFAP immunostaining (Figure 1D, G). Filamentous structures of EGFP-tagged GFAP could be colocalized with nestin and vimentin filaments in the transfected cells (Figure 1E, F, H, I). From our observations, the organization of endogenous nestin and vimentin filaments in C6 cells was not significantly altered by transient transfection with pEGFP-GFAP construct.

\section{Establishment of EGFP-GFAP stable cell line}

To clarify the long-term effect of overexpressed EGFP-tagged GFAP in C6 cells, we established the C6-EGFP-GFAP stable cell line. The expression of pEGFP-GFAP as well as the control pEGFP in stable clones was constitutive, and the green fluorescence of live cells could be observed under an inverted fluorescent microscope (Figure 2). In the control study of pEGFP, overexpression of EGFP could be found not only in the cytoplasm but also in the nucleus of the C6-EGFP cells (Figure 2A, B). Nevertheless, constitutively expressed EGFP-tagged GFAP was mainly found in the cytoplasm of C6-EGFP-GFAP cells (Figure $2 \mathrm{C}, \mathrm{D}$ ). Both C6-EGFP and C6-EGFP-GFAP cells bore slightly elongated processes and were not significantly different from each other.

Expression patterns of IF proteins and small heat shock protein $\alpha B$-crystallin in C6, C6-EGFP, and C6-EGFP-GFAP cells before and after heat shock treatment

Western blot analysis showed that the expression patterns of the IF proteins nestin, vimentin, GFAP, and small heat shock protein $\alpha \mathrm{B}$-crystallin in $\mathrm{C} 6$, C6-EGFP, and C6-EGFP-GFAP cells before heat shock treatment (Figure 3A). Expression levels of nestin among non-transfected C6, transfected C6EGFP, and C6-EGFP-GFAP cells were not significantly different. However, the level of vimentin in C6-EGFP-GFAP cells was lower than that in C6 and C6-EGFP cells. As we expected, EGFP-tagged GFAP protein could be detected as a molecular weight of approximately $75 \mathrm{kDa}$ in C6EGFP-GFAP cells. Endogenous GFAP could be also detected in C6, C6-EGFP, and C6-EGFPGFAP cells. We also examined the expression of small heat shock protein $\alpha \mathrm{B}$-crystallin in C6, C6EGFP, and C6-EGFP-GFAP cells. A basal level of $\alpha \mathrm{B}$-crystallin could be detected in $\mathrm{C} 6$ cells, whereas the level of this protein was slightly increased in C6-EGFP cells, and significantly enhanced in the C6-EGFP-GFAP cells.

After sublethal heat shock treatment at $42{ }^{\circ} \mathrm{C}$, we also examined the protein levels in $\mathrm{C} 6$, 


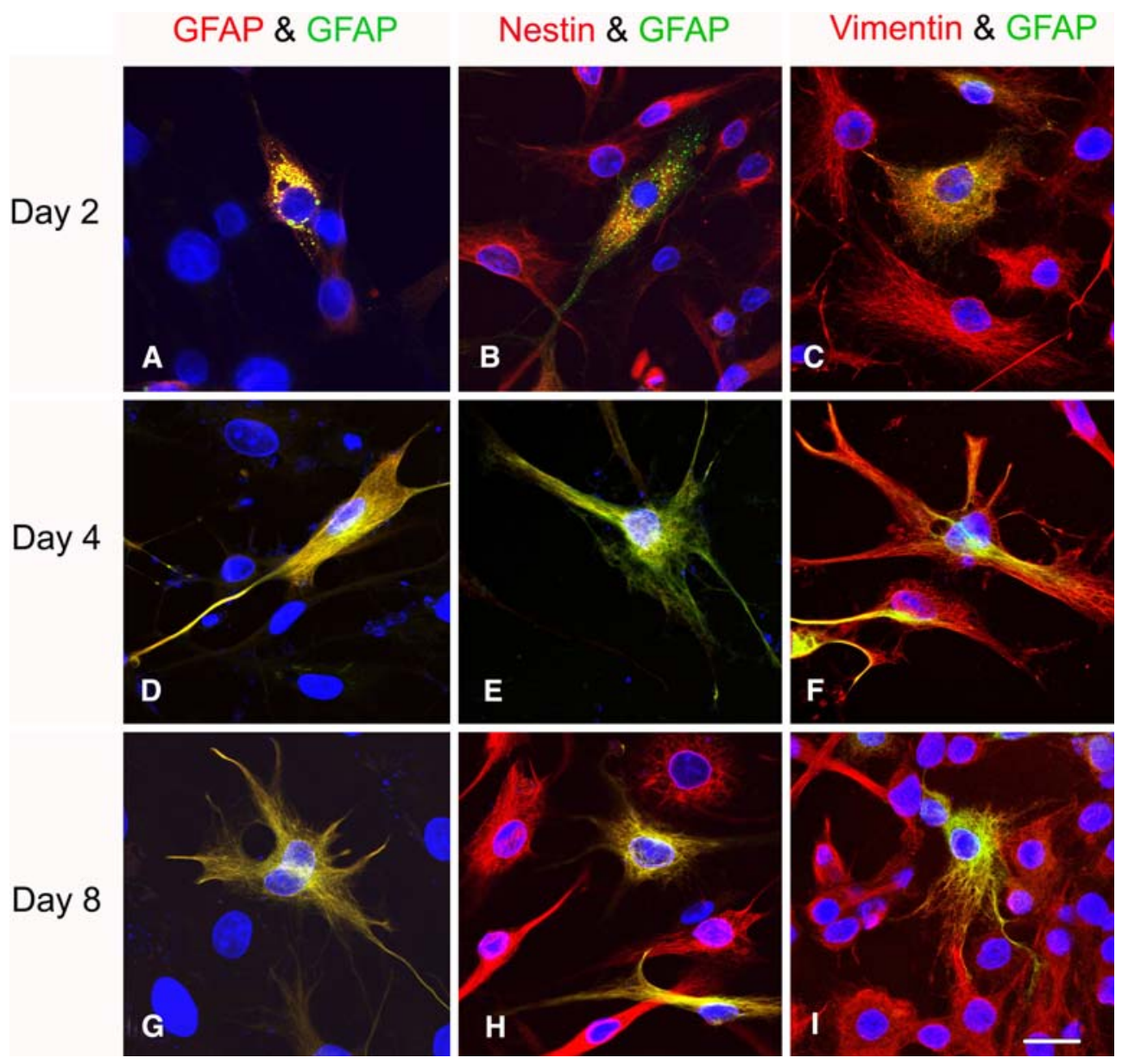

Figure 1. EGFP-tagged GFAP and its IF partners in C6 cells transiently transfected with pEGFP-GFAP. EGFP-tagged GFAP forms punctate aggregations alone with some filaments, whereas nestin and vimentin reside in the cytoplasm as filamentous structures 2 days after transfection (A, B, and C). Nevertheless, colocalization patterns of EGFP-tagged GFAP with antibody labeled GFAP, nestin, and vimentin are observed as filamentous structures 4 and 8 days after transfection (D-H and I). Scale bar $=20 \mu \mathrm{m}$

C6-EGFP, and C6-EGFP-GFAP cells by Western blot (Figure 3B). The protein level of nestin was similar among the C6, C6-EGFP, and C6EGFP-GFAP cells after heat shock. EGFP-tagged GFAP could be detected in C6-EGFP-GFAP cells, and endogenous GFAP was enhanced in C6EGFP-GFAP cells after heat shock. The expression level of $\alpha \mathrm{B}$-crystallin in C6 cells was low, whereas it was enhanced in C6-EGFP cells and C6-EGFP-GFAP cells, especially in C6-EGFPGFAP cells in this study. Besides, the slight proteolytic degradation of alpha-B-crystallin could be found in the C6-EGFP and in the C6-EGFPGFAP cells after heat shock treatment. According to our Western blot analysis, the expression of
GFAP as well as that of $\alpha \mathrm{B}$-crystallin was significantly affected in C6-EGFP-GFAP cells after heat shock treatment.

\section{Heat shock treatment and immunocytochemical patterns of IF proteins in C6-EGFP-GFAP cells}

To determine the organization and the distribution of IF proteins in C6-EGFP-GFAP cells, we investigated the cellular patterns of nestin, vimentin, and GFAP via the immunocytochemical approach (Figure 4). Nestin and vimentin organized into filamentous structure in C6EGFP-GFAP cells (Figure 4A, B), but the organization pattern of GFAP was a subtle filamentous 

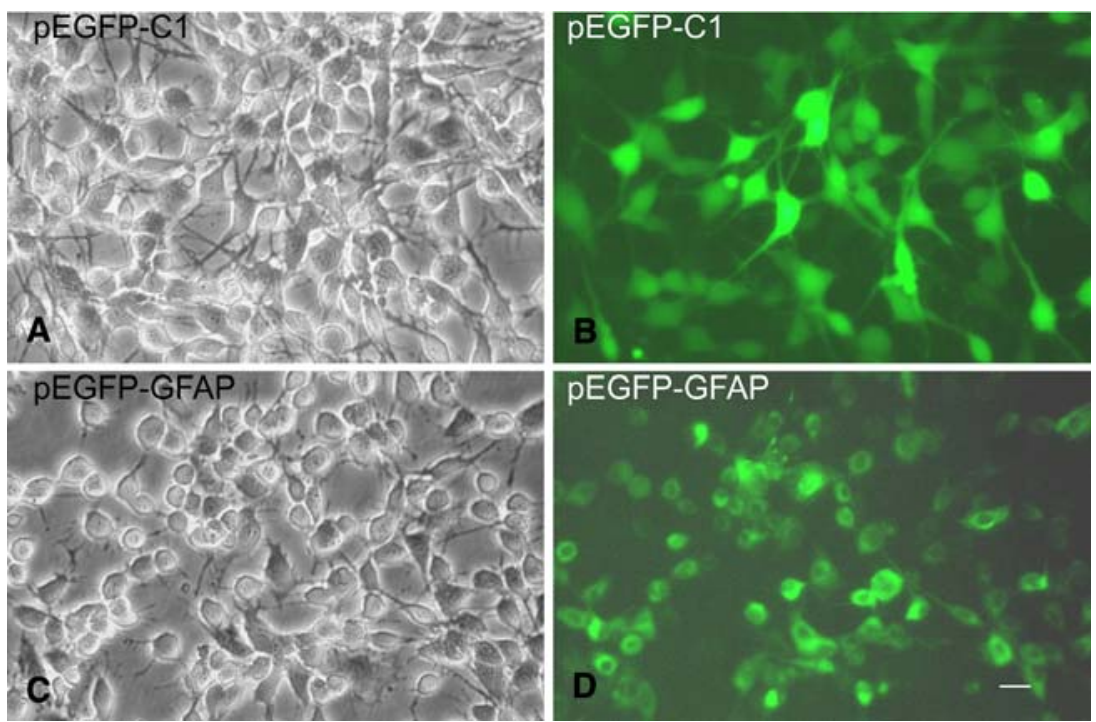

Figure 2. Stable cell clones established from C6 cells transfected with pEGFP-C1 and pEGFP-GFAP. Stable C6 cell clones transfected with pEGFP-C1 or pEGFP-GFAP are demonstrated by phase-contrast and live fluorescent images. Constitutively expressed EGFP can be observed in one of the C6-EGFP control groups (A and B), whereas the green fluorescence of EGFP-tagged GFAP is mainly found in the cytoplasm of C6-EGFP-GFAP cells (C and D). Scale bar $=200 \mu \mathrm{m}$.

pattern (Figure 4C). We also examined the correlation between thermal stress and IF organization in this study. After $1.5 \mathrm{~h}$ heat shock treatment at $42{ }^{\circ} \mathrm{C}$ followed by $16 \mathrm{~h}$ recovery, the organization patterns of nestin and vimentin were not significantly changed (Figure 4D, E). Interestingly, the filamentous pattern of GFAP was organized into clear bundles in heat shock treated cells (Figure 4F). Statistical analysis of GFAP bundlebearing cells versus total cell population also indicated that GFAP attempted to form IF bundles after heat shock treatment (Figure 4G).

\section{Immunocytochemical patterns of small heat shock protein $\alpha B$-crystallin in C6, C6-EGFP, and C6-EGFP-GFAP cells}

To study the distribution of small heat shock protein $\alpha \mathrm{B}$-crystallin in cells, we also checked on the immunocytochemical patterns of $\alpha \mathrm{B}$-crystallin in C6, C6-EGFP, and C6-EGFP-GFAP cells (Figure 5). $\alpha \mathrm{B}$-crystallin could be detected very weakly in both control and heat shock-treated C6 cells (Figure 5A, B). In C6-EGFP cells, $\alpha \mathrm{B}$ crystallin could be found in the cytoplasm and in the nucleus (Figure 5C). Nevertheless, positive immunostaining signals of $\alpha \mathrm{B}$-crystallin could be detected mainly in the nucleus of C6-EGFP cells after heat shock treatment (Figure 5D). The distribution of $\alpha \mathrm{B}$-crystallin was diffused and dispersed in the cytoplasm of C6-EGFP-GFAP cells before heat shock treatment (Figure 5E), yet redistribution of $\alpha \mathrm{B}$-crystallin was found in the filamentous structure in the cytoplasm of C6EGFP-GFAP cells after challenging with heat shock (Figure 5F). From this morphological observation, we also found that the expression level of $\alpha \mathrm{B}$-crystallin was enhanced in heat-shock treated C6-EGFP-GFAP cells.

\section{Colocalization of $\alpha B$-crystallin and GFAP in C6-EGFP-GFAP cells}

From the immunocytochemical evidence, we found that the expression of $\alpha \mathrm{B}$-crystallin was enhanced in C6-EGFP-GFAP cells. In order to understand the correlation between $\alpha \mathrm{B}$-crystallin and GFAP, we also investigated the double immunostaining patterns of $\alpha \mathrm{B}$-crystallin and GFAP in C6-EGFP-GFAP cells by laser confocal microscopy. A positive $\alpha \mathrm{B}$-crystallin immunostaining signal in a diffused pattern was seen within the C6-EGFP-GFAP cells (Figure 6A), yet after heat shock, $\alpha \mathrm{B}$-crystallin could be found mostly in the intermediate filament network of treated cells (Figure 6B). GFAP was distributed as a fine 

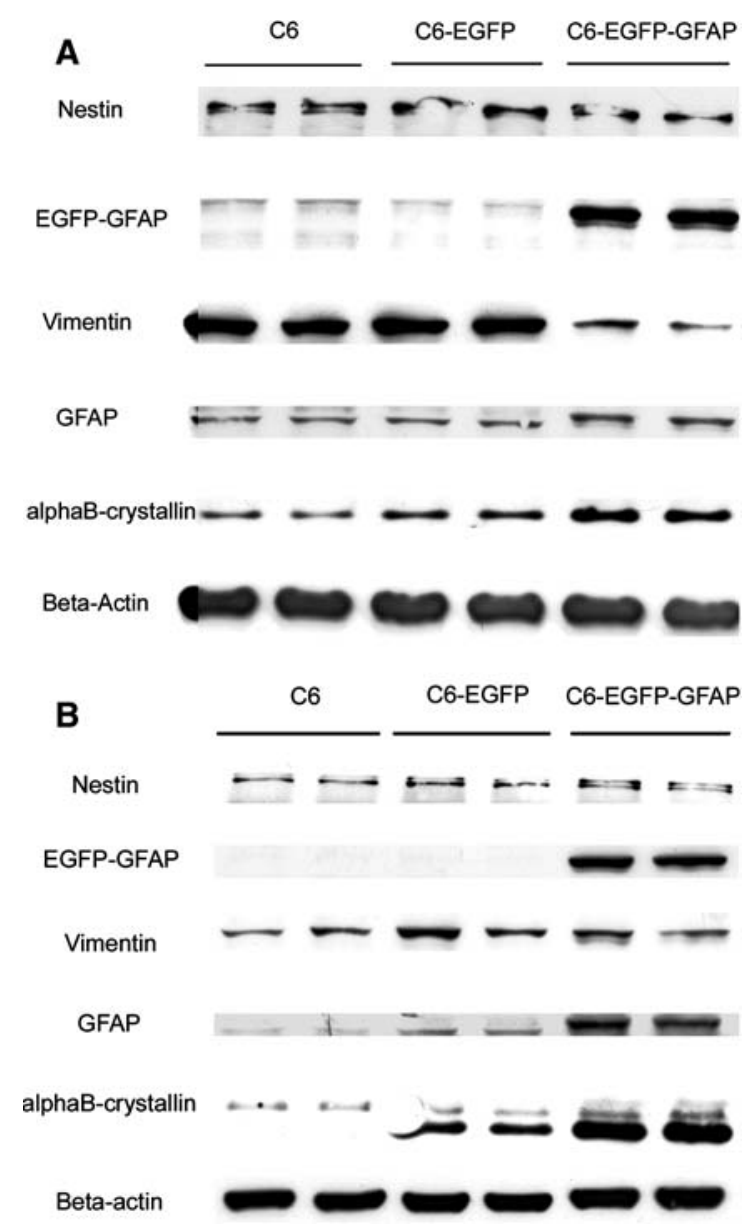

Figure 3. Western blots of IF proteins and small heat shock proteins $\alpha$ B-crystallin in C6, C6-EGFP, and C6-EGFP-GFAP cells before and after heat-shock treatments. Before heat shock treatments (A), protein levels of nestin and endogenous GFAP are similar among C6, C6-EGFP, and C6EGFP-GFAP cells. The $75 \mathrm{kDa}$ EGFP-tagged GFAP can be detected, yet the protein level of vimentin is reduced in C6-EGFP-GFAP cells. Notice that the expression of small heat shock protein $\alpha \mathrm{B}$-crystallin is enhanced in C6-EGFP and C6-EGFP-GFAP cells, especially in C6-EGFP-GFAP cells (A). After heat shock treatment (B), protein levels of nestin and vimentin show similar patterns among C6, C6-EGFP, and C6-EGFP-GFAP cells. Overexpressed EGFP-tagged GFAP can be detected in C6-EGFP-GFAP cells, and low levels of endogenous GFAP can be detected as weak bands in C6, C6-EGFP, and C6-EGFP-GFAP cells. Notice that the expression levels of $\alpha \mathrm{B}$-crystallin are prominently enhanced in C6-EGFP-GFAP cells (B).

filamentous structure and partially diffused in the C6-EGFP-GFAP cells before heat shock, whereas GFAP organized into filamentous bundles after heat challenge (Figure 6C, D). Merged panels of $\alpha \mathrm{B}$-crystallin and GFAP also indicated that $\alpha \mathrm{B}$-crystallin partially colocalized with GFAP in the C6-EGFP-GFAP cells, especially in the filamentous bundles after heat shock treatment (Figure $6 \mathrm{E}, \mathrm{F})$. Ultrastructural patterns of C6, C6-EGFP,
and C6-EGFP-GFAP cells

To study the intracellular effects of thermal stress, we also inspected the ultrastructural patterns of C6-EGFP and C6-EGFP-GFAP cells. In C6EGFP cells, 10-nm intermediate filaments were found in the cytoplasm, and other organelles such as the mitochondria looked normal (Figure 7A). After heat shock treatment, a few damaged mitochondria with swelling patterns could be found in C6-EGFP cells (Figure 7B). The arrangement of intermediate filaments in C6-EGFP-GFAP was not particularly changed before heat shock (Figure $7 \mathrm{C}$ ). But intermediate filaments were reorganized into more compact patterns (arrow), and a few multi-layers of autophagosomes were also observed in heat shock treated C6-EGFP-GFAP cells (Figure 7D). From this ultrastructural observation, we double-confirmed the filamentous bundling pattern of GFAP observed by immunofluorescence in heat shock treated C6EGFP-GFAP cells.

\section{Discussion}

GFAP is able to coassemble with nestin and vimentin

In the study of transient transfection, we found that EGFP-tagged GFAP formed punctate aggregations in the cytoplasm of $\mathrm{C} 6$ cells right after transfection, but gradually a filamentous structure was observed. According to our immunocytochemical observations, the organization of EGFP-tagged GFAP in C6 cells did not alter the cytoarchitecture of endogenous nestin and vimentin filaments, although the organization pattern of EGFP-tagged GFAP in C6 cells was dynamic. The colocalization pattern also showed that EGFP-tagged GFAP could be incorporated into the nestin and vimentin network. These findings suggested that our pEGFP-GFAP construct with full-length rat GFAP fused at its $\mathrm{N}$-terminus to EGFP was efficient to form an intermediate filament network, and showed the 

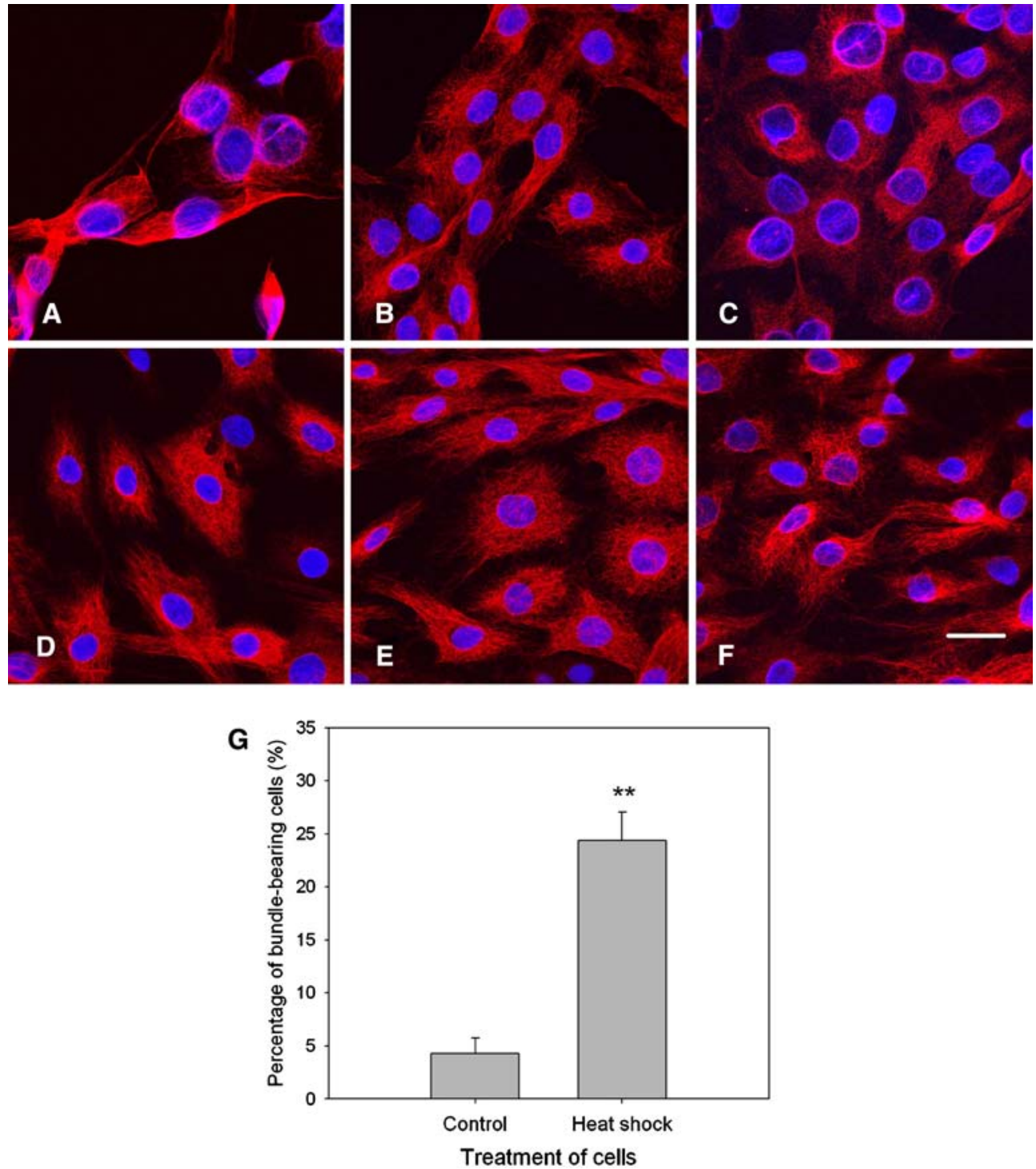

Figure 4. Organization patterns of IF proteins in C6-EGFP-GFAP cells after heat shock treatment. Immunocytochemical patterns of nestin (A and D), vimentin (B and E), and GFAP (C and F) before and after heat shock in treated C6-EGFP-GFAP cells are shown. Organization patterns of nestin and vimentin are seen as filamentous structures in control (A and B) and heat shock treated (D and E) C6-EGFP-GFAP cells. However, GFAP has formed some fine filaments in the cytoplasm of C6-EGFP-GFAP cells before heat shock (C). After heat shock, GFAP is organized into clear IF bundles (F). GFAP bundle-bearing cells were counted under a microscope for statistical analysis. In each case, we randomly chose three fields under a $40 \times$ object lens of a fluorescent microscope. In each field, 300 cells labeled with Hoechst 33342 were counted and then GFAP bundle-bearing cells labeled with GFAP antibody were counted in the same population. Twenty-seven fields from 9 coverslips in each group were counted and the percentage of GFAP bundle-bearing cells in C6-EGFP-GFAP is shown $(\mathrm{G})$. Notice that the percentage of GFAP bundle-bearing cells in the heat shock group is significantly greater than that in control group ( $t$-test, $p<0.001)$. Scale bar $=20 \mu \mathrm{m}$.

same ability as wild type GFAP to coassemble with nestin and vimentin. IF protein binding partnerships of nestin, vimentin, and GFAP could be observed in primary astrocyte cultures and reactive astrocytes [5]. Previous studies using overexpressed EGFP-tagged vimentin also showed a coassembly pattern with endogenous vimentin filaments in SW13 vim ${ }^{+}$cells and NIH3T3 cells [27], which suggested that EGFP fused with the N-terminus of type III IF proteins was efficient to form IF in the presence of endogenous IF proteins. 

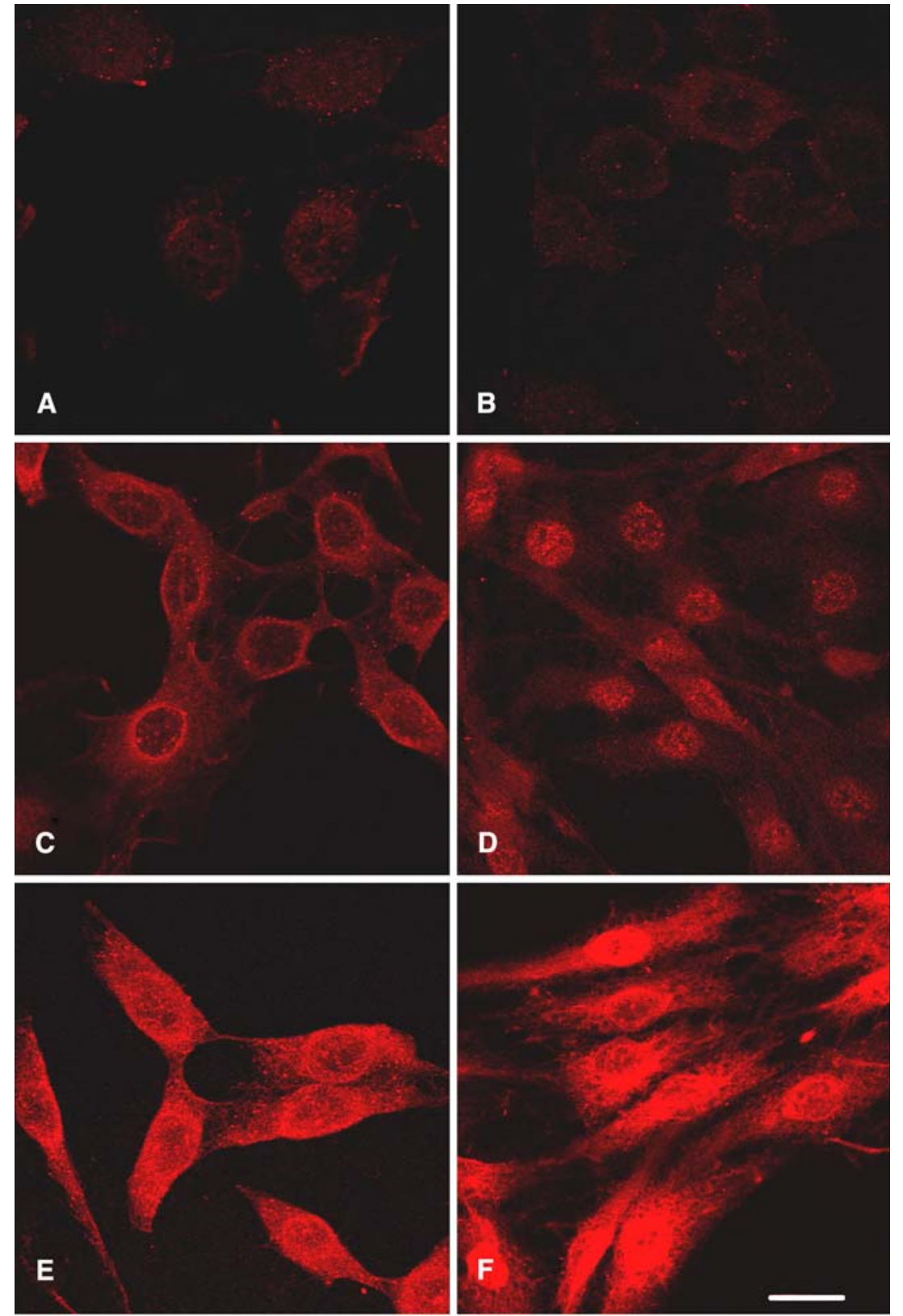

Figure 5. The distribution of $\alpha \mathrm{B}$-crystallin in control and heat shock treated C6, C6-EGFP, and C6-EGFP-GFAP cells. $\alpha \mathrm{B}-\mathrm{crystallin}$ immuno-positive signals can be observed very weakly in control and in heat shock treated C6 cells (A and B). The distribution of $\alpha \mathrm{B}$-crystallin in C6-EGFP cells is mainly in the cytoplasm before heat shock, whereas it resides prominently in the nucleus after heat shock (C and D). In C6-EGFP-GFAP cells, $\alpha \mathrm{B}$-crystallin is distributed as diffused patterns in the whole cell before heat shock (E). $\alpha \mathrm{B}$-crystallin is relocated onto the filamentous structures after heat shock treatment $(\mathrm{F})$. Scale bar $=20 \mu \mathrm{m}$.

The up-regulation effect of $\alpha B$-crystallin was more significant in GFAP overexpressed cells

It has been reported that GFAP can interact with some small heat shock proteins, such as
HSP27 and $\alpha$ B-crystallin. These small heat shock proteins might act as molecular chaperones to modulate the distribution of GFAP [25]. Furthermore, $\alpha \mathrm{B}$-crystallin is more efficient in dealing with GFAP organization than HSP27 [28]. 

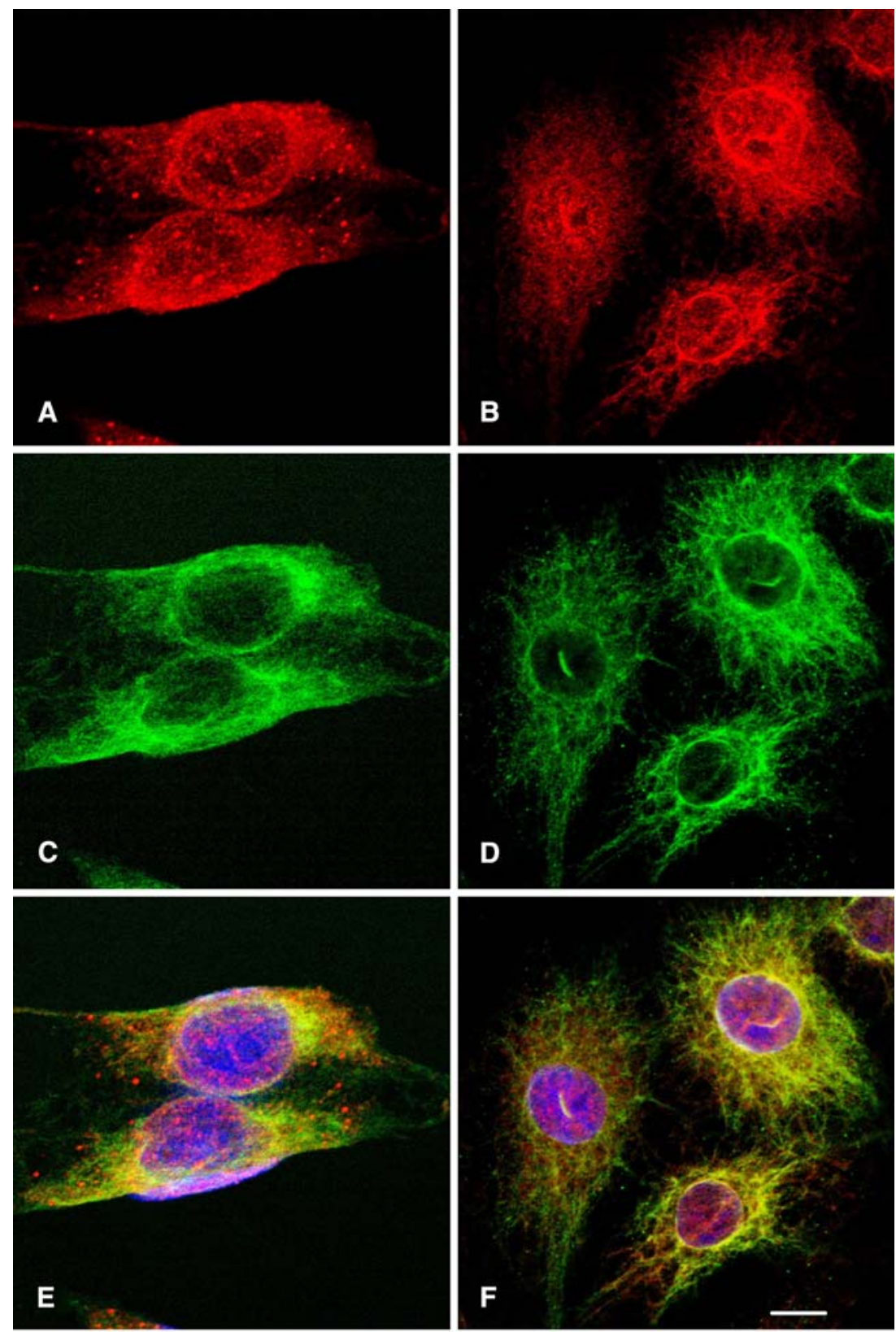

Figure 6. Colocalization patterns of $\alpha$ B-crystallin and GFAP in C6-EGFP-GFAP cells. The relationship between distribution patterns of $\alpha \mathrm{B}$-crystallin and GFAP is demonstrated by immunocytochemistry. $\alpha \mathrm{B}$-crystallin is redistributed from a diffused pattern (A) to a filamentous-like pattern (B) after heat shock treatment (B). GFAP is organized into a fine filamentous pattern in C6-EGFP-GFAP before heat shock (C) rather than the IF bundles in the heat shock group (D). Merged images show colocalization patterns of $\alpha$ B-crystallin and GFAP (E and F). Scale bar $=8 \mu \mathrm{m}$.

The correlation of $\alpha \mathrm{B}$-crystallin and GFAP can be traced to the finding from research on Alexander disease (ALX) [29, 30]. ALX, a rare disorder of the central nervous system, was found to be associated with mutations of the
GFAP gene [31]. The hallmark of Alexander disease is Rosenthal fibers (RFs), which are eosinophilic, round or oblong bodies within the cytoplasm of astrocytes [30, 32, 33]. The composition of Rosenthal fibers includes GFAP and 

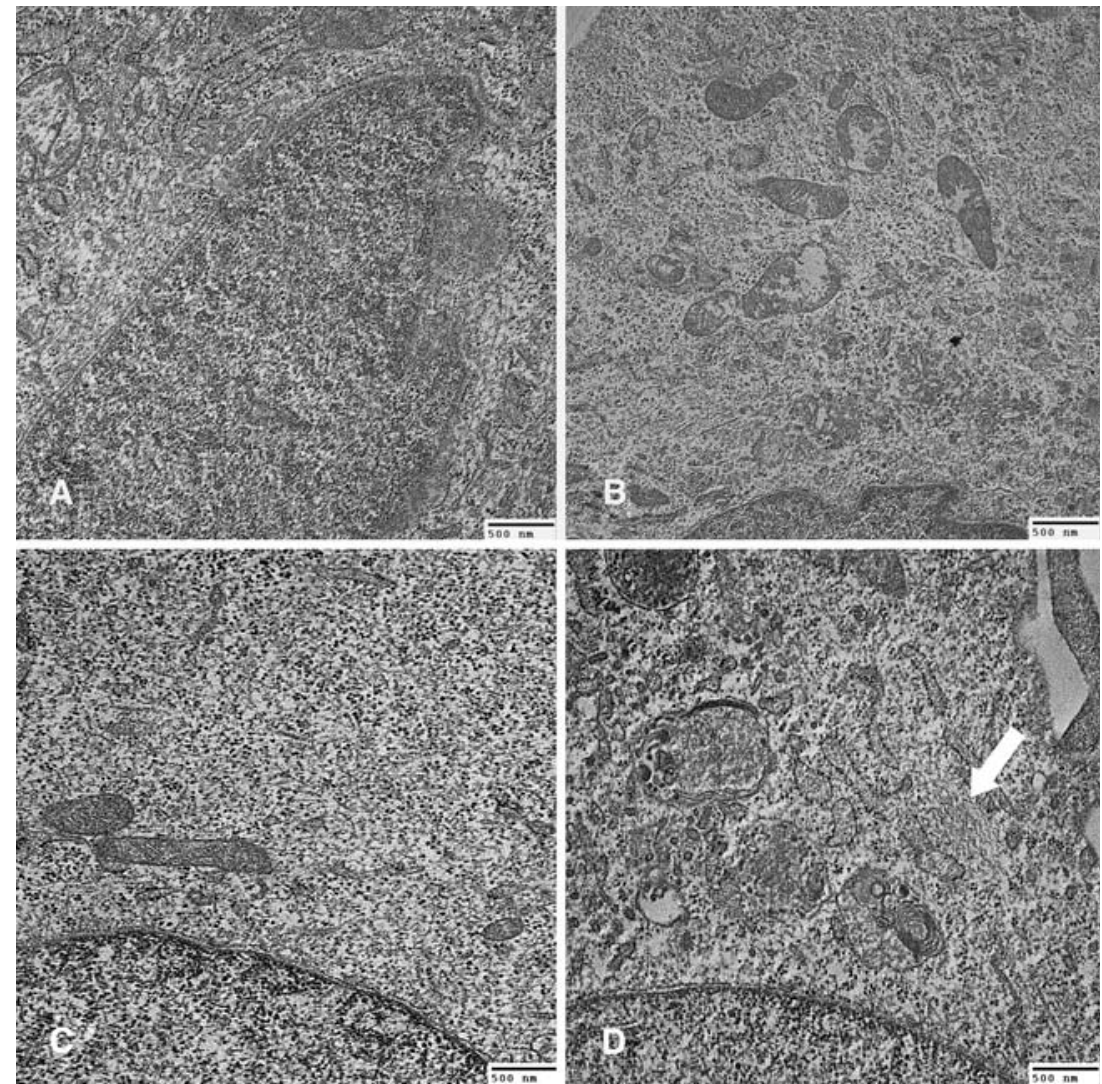

Figure 7. Ultrastructural patterns of C6-EGFP and C6-EGFP-GFAP cells after heat shock treatment. Some intermediate filaments can be seen around the perinuclear region in C6-EGFP cells before heat shock (A). Abnormal mitochondria with swelling patterns are observed in heat shock treated C6-EGFP cells (B). More intermediate filaments are observed in the cytoplasm in C6-EGFP-GFAP cells (C). The bundling pattern of IF can be seen after heat shock (D, arrow).

the small heat shock proteins $\alpha \mathrm{B}$-crystallin, hsp27, and ubiquitin [30]. A transgenic study of mice carrying an additional human GFAP gene showed that GFAP and $\alpha \mathrm{B}$-crystallin formed Rosenthal fibers in astrocytes [34]. In the present study, the level of $\alpha \mathrm{B}$-crystallin was slightly increased in C6-EGFP cells and this may be a response to the overexpression of EGFP driven by CMV promoter. EGFP was overexpressed in C6-EGFP cells and endogenous $\alpha \mathrm{B}$ crystallin could act as the molecular chaperone to prevent the formation of protein aggregation within the cell [21]. Our observations that the up-regulation effect of $\alpha \mathrm{B}$-crystallin was more significant in C6-EGFP-GFAP cells than in C6EGFP cells reveals that $\alpha \mathrm{B}$-crystallin may participate in the regulation of GFAP pattern formation.
The organization of GFAP is more easily affected than that of nestin and vimentin under thermal stress

Thermal stress is a kind of physiological stress which may induce responses in cells, such as reorganizing the cytoskeleton and turning on heat shock response genes. Cells may respond by trying to accommodate the change in the extracellular microenviroment and maintain homeostasis. Previous study has proved that GFAP reorganizes into a bundling pattern around the nucleus in U373MG astrocytoma cells after heat shock treatment [25]. It was also demonstrated that GFAP reorganizes into a compact pattern around the perinuclear region of human optic nerve head astrocytes exposed to elevated hydrostatic pressure in vitro [35]. In our experiment, we found that GFAP filaments reorganized into clear IF bundles 
in C6-EGFP-GFAP cells after heat shock, whereas the expression of endogenous GFAP was also slightly enhanced. This result is similar to previous observations [25]. Interestingly, the organization and distribution of nestin and vimentin filaments in C6-EGFP-GFAP were not altered after heat shock treatment. It seems that organization of GFAP is more easily affected than that of nestin and vimentin under thermal stress. From our observations, it could be suggested that IF bundles can provide stronger mechanical resistance when cells deal with certain kinds of stress. GFAP may be the best candidate among the IF proteins in glial cells to cope with a heat shock challenge.

$\alpha B$-crystallin may play a role in the maintenance of normal IF architecture before and after heat shock treatment

After GFAP reorganized into IF bundles under thermal stress, we found that small heat shock protein $\alpha \mathrm{B}$-crystallin was present on these bundles. The protein expression level of $\alpha \mathrm{B}$-crystallin was also significantly enhanced in C6-EGFP-GFAP cells after heat shock treatment. In the Western blot, an extra band with the higher molecular weight for $\alpha \mathrm{B}$-crystallin could also be detected after heat shock. We expected this might be due to the phosphorylation of the protein. This could be supported by a previous study in which the phsphorylation of $\alpha \mathrm{B}$-crystallin was enhanced in U373MG cells after heat shock [36]. An association of $\alpha \mathrm{B}$-crystallin with the vimentin filaments in NIH 3 T3 cells was also found after a $43{ }^{\circ} \mathrm{C}$ heat shock challenge [37]. Moreover, the association of $\alpha \mathrm{B}$-crystallin with GFAP was maintained during stress-induced bundling of intermediate filament in U373MG cells [25]. From these observations, we propose that the redistribution of $\alpha \mathrm{B}$-crystallin in C6-EGFP-GFAP cells after heat shock results from the character of the small heat shock protein. It has been reported that $\alpha \mathrm{B}$-crystallin and some other small heat shock proteins are involved in the maintenance of normal IF architecture [38]. According to our ultrastructural observations, thermal stress-induced bundling of GFAP filaments is due to the accumulation of $10 \mathrm{~nm}$ intermediate filaments, which can be categorized as the formation of a mild IF aggregation. The association of $\alpha \mathrm{B}$-crystallin with these IF bundles may prevent further severe IF aggregation after heat shock. This viewpoint is also supported by a previous study which showed that $\alpha \mathrm{B}$-crystallin acted as an IF debundling protein to regulate the organization of intermediate filaments in vitro [28]. Therefore, we could make a conclusion that $\alpha \mathrm{B}$ crystallin plays the role of an IF debundling factor but not a soluble GFAP regulator in the heat shock experiment and the role of $\alpha \mathrm{B}$-crystallin on GFAP may be different before and after heat shock treatment.

In summary, we found that overexpression of EGFP-tagged GFAP resulted in the formation of a fine filamentous structure and did not alter the arrangement of nestin and vimentin filaments; furthermore it also enhanced the protein level of small heat shock protein $\alpha \mathrm{B}$-crystallin in transfected $\mathrm{C} 6$ cells. These findings suggest that $\alpha \mathrm{B}-$ crystallin might regulate the distribution of overexpressed EGFP-tagged GFAP. But we still cannot rule out the participation of some other proteins involved in the organization of the IF network, such as cytoskeletal crosslinker proteins, intermediate filament associated proteins (IFAPs), and some small heat shock proteins. In our experiment, we demonstrated that overexpression of EGFP-tagged GFAP up-regulates the protein level of $\alpha \mathrm{B}$-crystallin. We also discussed that the function of $\alpha \mathrm{B}$-crystallin on GFAP may be different before and after heat shock. The heat shock treatment induced the reorganization of GFAP filaments into IF bundles in C6-EGFP-GFAP cells, which may result from a protective effect of $\alpha \mathrm{B}$-crystallin when dealing with thermal stress. These observations may help us understand the function of $\alpha \mathrm{B}$-crystallin on GFAP organization in glial cells under stress, in tumors, and in neurodegenerative diseases.

\section{Acknowledgements}

We would like to thank Dr. R Liem (Columbia University, New York, USA) for the gift of GFAP clone. We are grateful to Dr. H.-Y. Yang (Institute of Molecular and Cellular Biology, National Taiwan University, Taipei, Taiwan) for valuable suggestions and for the gift of C6 cells. This work was supported by a grant to C.-L. Chien (NSC 94-2320-B-002-051)) from the National Science Council, Taiwan. Facilities provided by grants from the Ministry of Education, 
Taiwan to J.-Y. Lin (Program for Promoting Academic Excellence of Universities 89-B-FA011-4) and to the Center for Genomic Medicine in National Taiwan University (93-K027) are also acknowledged.

\section{References}

1. Eng L.F., Gerstl B. and Vanderhaeghen J.J., A study of proteins in old multiple sclerosis plaques. Trans. Am. Soc. Neurochem. 1: 42, 1970.

2. Eng L.F., Ghirnikar R.S. and Lee Y.L., Glial fibrillary acidic protein: GFAP - thirty-one years (1969-2000). Neurochem. Res. 25: 1439-1451, 2000.

3. Inagaki M., Nakamura Y., Takeda M., Nishimura T. and Inagaki N., Glial fibrillary acidic protein: dynamic property and regulation by phosphorylation. Brain Pathol. 4: 239243, 1994.

4. Rutka J., Murakami M., Dirks P., Hubbard S., Becker L., Fukuyama K., Jung S., Tsugu A. and Matsuzawa K., Role of glial filaments in cells and tumors of glial origin: a review. J. Neurosurg. 87: 420-430, 1997.

5. Eliasson C., Sahlgren C., Berthold C.H., Stakeberg J., Celis J.E., Betsholtz C., Eriksson J.E. and Pekny M., Intermediate filament protein partnership in astrocytes. J. Biol. Chem. 274: 23996-24006, 1999.

6. Eng L.F. and Ghirnikar R.S., GFAP and astrogliosis. Brain Pathol. 4: 229-237, 1994.

7. Frisén J., Johansson C.B., Török C., Risling M. and Lendahl U., Rapid, widespread, and longlasting induction of nestin contributes to the generation of glial scar tissue after CNS injury. J. Cell Biol. 131: 453-464, 1995.

8. Liedtke W., Edelman W., Bieri P.L., Chiu F.C., Cowan N.J., Kucherlapati R. and Raine C.S., GFAP is necessary for the integrity of CNS white matter architecture and longterm maintenance of myelination. Neuron 17: 607-615, 1996.

9. Shibuki K., Gomi H., Chen L., Bao S., Kim J.J., Wakatsuki H., Fujisaki T., Fujimoto K., Katoh A., Ikeda T., Chen C., Thompson R.F. and Itohara S., Deficient cerebellar long-term depression, impaired eyeblink conditioning, and normal motor coordination in GFAP mutant mice. Neuron 16: 587-599, 1996.

10. McCall M.A., Gregg R.G., Behringer R.R., Brenner M., Delaney C.L., Galbreth E.J., Zhang C.L., Pearce R.A., Chiu S.Y. and Messing A., Targeted deletion in astrocyte intermediate filament (GFAP) alters neuronal physiology. Proc. Natl. Acad. Sci. USA 93: 6361-6366, 1996.

11. Pekny M., Eliasson C., Chien C.L., Kindblom L.G., Liem R., Hamberger A. and Betsholtz C., GFAP-deficient astrocytes are capable of stellation in vitro when cocultured with neurons and exhibit a reduced amount of intermediate filaments and an increased cell saturation density. Exp. Cell Res. 239: 332-343, 1998.

12. Weinstein D.E., Shelanski M.L. and Liem R.K., Suppression by antisense mRNA demonstrates a requirement for the glial fibrillary acidic protein in the formation of stable astrocytic processes in response to neurons. J. Cell Biol. 112: 1205-1213, 1991.

13. Gomi H., Yokoyama T., Fujimoto K., Ideka T., Katoh A., Itoh T. and Itohara S., Mice devoid of the glial fibrillary acidic protein develop normally and are susceptible to scrapie prions. Neuron 14: 29-41, 1995.

14. Pekny M., Levéen P., Pekna M., Eliasson C., Berthold C.H., Westermark B. and Betsholtz C., Mice lacking glial fibrillary acidic protein display astrocytes devoid of intermediate filaments but develop and reproduce normally. EMBO J. 14: 1590-1598, 1995.

15. Koyama Y. and Goldman J.E., Formation of GFAP cytoplasmic inclusions in astrocytes and their disaggregation by $\alpha$ B-crystallin. Am. J. Pathol. 154: 1563-1572, 1999.

16. Iwaki T., Kume-Iwaki A., Liem R.K. and Goldman J.E., $\alpha \mathrm{B}$-crystallin is expressed in non-lenticular tissue and accumulates in Alexander's disease. Cell 57: 71-78, 1989.

17. Bhat S.P. and Nagineni C.N., $\alpha B$ subunit of lens-specific protein a-crystallin is present in other ocular and nonocular tissue. Biochem. Biophys. Res. Commun. 158: 319325, 1989.

18. Dubin R.A., Wawrousek E.F. and Piatigorsky J., Expression of the murine aB-crystallin gene is not restricted to the lens. Mol. Cell Biol. 9: 1083-1091, 1989.

19. Aoyama A., Frohli E., Schafere R. and Klemenz R., $\alpha \mathrm{B}$-crystallin expression in mouse NIH3T3 fibroblasts: glucocorticoid responsiveness and involvement in thermal protection. Mol. Cell Biol. 13: 1824-1835, 1993.

20. Head M.W., Corbin E. and Goldman J.E., Coordinate and independent regulation of $\alpha \mathrm{B}$-crystallin and HSP27 expression in response to physiological stress. J. Cell Physiol. 159: $41-50,1994$

21. Horwitz J., $\alpha$-Crystallin can function as a molecular chaperone. Proc. Natl. Acad. Sci. USA 89: 10449-10453, 1992.

22. Bennardini F., Wrzosek A. and Chiesi M., $\alpha$ B-crystallin in cardiac tissue: association with actin and desmin filaments. Circ. Res. 71: 288-294, 1992.

23. Nicholl I.D. and Quinlan R.A., Chaperone activity of $\alpha$-crystallins modulates intermediate filament assembly. EMBO J. 13: 945-953, 1994.

24. Wisniewski T. and Goldman J.E., $\alpha$ B-crystallin is associated with intermediate filaments in astrocytoma cells. Neurochem. Res. 23: 385-392, 1998.

25. Perng M.D., Cairns L., van den IJssel P., Prescott A., Hutcheson A.M. and Quinlan R.A., Intermediate filament interactions can be altered by HSP27 and alphaB-crystallin. J. Cell Sci. 112: 2099-2112, 1999.

26. Johnson A.B., Alexander disease: a review and the gene. Int. J. Dev. Neurosci. 20: 391-394, 2002.

27. Ho C.L., Martys J.L., Mikhailov A., Gundersen G.G. and Liem R.K., Novel features of intermediate filament dynamics revealed by green fluorescent protein chimeras. J. Cell Sci. 111: 1767-1778, 1998.

28. Head M.W., Hurwitz L., Kegel K. and Goldman J.E., AlphaB-crystallin regulates intermediate filament organization in situ. Neuroreport 11: 361-365, 2000.

29. Alexander W.S., Progressive fibrinoid degeneration of fibrillary astrocytes associated with mental retardation in a hydrocephalic infant. Brain 72: 373-381, 1949.

30. Herndon R.M., Rubinstein L.J. and Freeman J.M., Light and electron microscopic observations on Rosenthal fibers in Alexander's disease and in multiple sclerosis. J. Neuropathol. Exp. Neurol. 29: 524-551, 1970.

31. Brenner M., Johnson A.B., Boespflug-Tanguy O., Rodriguez D., Goldman J.E. and Messing A., Mutations in GFAP, encoding glial fibrillary acidic protein, are associated with Alexander disease. Nat. Genet. 27: 117-120, 2001 . 
32. Grcevic N. and Yates P.O., Rosenthal fibers in tumors of the central nervous system. J. Pathol. Bacteriol. 73: 467-472, 1957.

33. Chin S.S. and Goldman J.E., Glial inclusions in CNS degenerative diseases. J. Neuropathol. Exp. Neurol. 55: 499-508, 1996.

34. Eng L.F., Lee Y.L., Kwan H., Brenner M. and Messing A., Astrocytes cultured from transgenic mice carrying the added human glial fibrillary acidic protein gene contain rosenthal fibers. J. Neurosci. Res. 53: 353-360, 1998.

35. Salvador-Silva M., Ricard C.S., Agapova O.A., Yang P. and Hernandez M.R., Expression of small heat shock proteins and intermediate filaments in the human optic nerve head astrocytes exposed to elevated hydrostatic pressure in vitro. J. Neurosci. Res. 66: 59-73, 2001.

36. Ito H., Kamei K., Iwamoto I., Inaguma Y., Nohara D. and Kato K., Phosphorylation-induced change of the oligomerization state of alpha B-crystallin. J. Biol. Chem. 276: 5346-5352, 2001.

37. Djabali K., deNechaud B., Landon F. and Portier M.M., alphaB-crystallin interacts with intermediate filaments in response to stress. J. Cell Sci. 110: 2759-2769, 1997.

38. Liang P. and MacRae T.H., Molecular chaperones and the cytoskeleton. J. Cell Sci. 110: 1431-1440, 1997. 